\title{
Ultraschnelle bildgebende Ramanspektroskopie an großflächigen Proben ohne schrittweise Abtastung
}

\author{
Elmar Schmälzlin, Benito Moralejo, Martin M. Roth \\ Leibniz-Institut für Astrophysik Potsdam (AIP), An der Sternwarte 16, 14482 Potsdam, Deutschland, \\ eschmaelzlin@aip.de
}

\begin{abstract}
Kurzbeschreibung:
Bildgebende Ramanspektrographen tasten eine Probe üblicherweise zeitaufwändig Punkt für Punkt ab. Selbst bei wenigen 100 Bildpunkten liegen die erforderlichen Messzeiten im Minuten- oder gar im Stundenbereich. Eine drastische Verkürzung der Messdauer lässt sich mit faserbündelgekoppelten Astronomiespektrographen erzielen. Um die knappen Beobachtungszeiten an Großteleskopen optimal ausnutzen zu können, wurden Spektrographensysteme entwickelt, die in einem einzigen Belichtungsvorgang tausende ortsaufgelöste Spektren eines zweidimensionalen Bildfeldes aufnehmen. Eine Übertragung dieser Technologie in die bildgebende Ramanspektroskopie ermöglicht eine deutlich schnellere Erstellung chemischer Karten. Da Bildfelder von mehreren Quadratmillimeter auf einmal erfasst werden können, ist diese Technik besonders interessant für die medizinische Diagnostik, z. B. für die Identifizierung von krebsverdächtigem Hautgewebe.
\end{abstract}

Schlagwörter: Bildgebende Ramanspektroskopie, Integrale-Feld-Spektroskopie, Faserbündel, Astronomiespektrograph.

\section{Einleitung}

Trifft auf eine chemische Probe monochromatisches Licht, so kommt es im Streulicht zu Farbverschiebungen, die mit den Schwingungsniveaus der Molekül- und Kristallstrukturen korrespondieren. Die auftretenden Ramanlinien bilden einen spektralen Fingerabdruck, mit dessen Hilfe man Molekül- oder Kristallstrukturen der Probe berührungs- und markerfrei identifizieren kann. Entdeckt wurde der zugrunde liegende Effekt bereits 1928 von dem Indischen Physiker Chandrasekhara Venkata Raman. Da Ramanlinien normalerweise aber extrem intensitätsschwach sind (nur etwa $1 \times 10^{-7}$ des gestreuten Lichtes ist Ramanstreuung), erlangte die Ramanspektroskopie zunächst nur wenig praktische Bedeutung. Einhergehend mit den Fortschritten bei der Laser- und Detektortechnik hat sich diese Situation allerdings in den letzten zehn Jahren grundlegend geändert. Besonders im Bereich der medizinischen Diagnostik und der Lebenswissenschaften sind mehr und mehr kommerzielle Ramanmikroskope anzutreffen, die sich heute neben den traditionellen Lichtund Fluoreszenzmikroskopen etablieren. Im Gegensatz zur klassischen Infrarot-Absorptionsspektroskopie, die ebenfalls chemische Substanzen über die energetische Lage ihrer Schwingungsniveaus identifiziert, funktioniert
Ramanspektroskopie auch in wässriger Umgebung, was im Hinblick auf biologische Proben von großem Vorteil ist. Mit entsprechenden Spektrographen lassen sich zwei- oder sogar dreidimensionale chemische Karten von Zellund Gewebeproben erstellen, die beispielsweise zur Identifizierung von krebsverdächtigen Hautpartien verwendet werden können [1]. Eine Vorstellung aktueller Methoden und Anwendungen aus dem Gebiet der bildgebenden Ramanspektroskopie findet sich in $[2,3]$. Übliche Ramanmikroskope tasten die Proben zeitaufwändig Schritt für Schritt ab. Die erforderlichen Messzeiten können bereits bei mehreren 100 Bildpunkten in den Stundenbereich gehen. Für routinemäßige Untersuchungen direkt am $\mathrm{Pa}$ tienten oder für sich verändernde Proben dauert so ein Verfahren viel zu lange. Abhilfe schafft die Integrale-Feld-Spektroskopie (IFS; Integral Field Spectroscopy), ein Verfahren aus der Astronomie. Diese Mehrkanal-Spektroskopie-Technik wurde in den letzten Jahrzehnten entwickelt, um Bilder lichtschwacher Galaxien in einem einzigen Belichtungsvorgang spektralund ortsaufgelöst zu erfassen und auf diese Weise die knappen Beobachtungszeiten an Großteleskopen optimal auszunutzen. Der derzeit leistungsstärkste Spektrograph "MUSE" (Multi Unit Spectroscopic Explorer) wurde im Frühjahr 2014 im „Very Large Telescope Ob- 
servatory in Paranal, Chile in Betrieb genommen [4]. Bildfelder des Himmels einer Größe von 1 arcmin $^{2}$ werden mit einer Auflösung von 90.000 Bildpunkten mit nur einem Belichtungsvorgang aufgenommen. In jedem der 90.000 Bildpunkte wird dabei das komplette Spektrum von 465 bis $930 \mathrm{~nm}$ mit einer Auflösung von etwa 0,25 nm erfasst. Im Rahmen eines Technologietransferprojektes wurde eine 25. MUSEEinheit mit einem Faserbündel gekoppelt und am AIP installiert. Ziel des Vorhabens ist es, die Tauglichkeit von IFS für die Erkennung von krebsverdächtigen Hautpartien zu untersuchen und dabei in Hinblick auf die Patientenfreundlichkeit die Messzeiten möglichste kurz zu halten.

\section{Versuchsaufbau}

Eine ausführliche Beschreibung der Spektrographeneinheit findet sich in $[4,5]$. Abb. 1 zeigt das Funktionsprinzip. In der Bildebene der Probe befindet sich die Erfassungseinheit für 400 Bildpunkte, d.h. eine Matrix aus $20 \times 20$

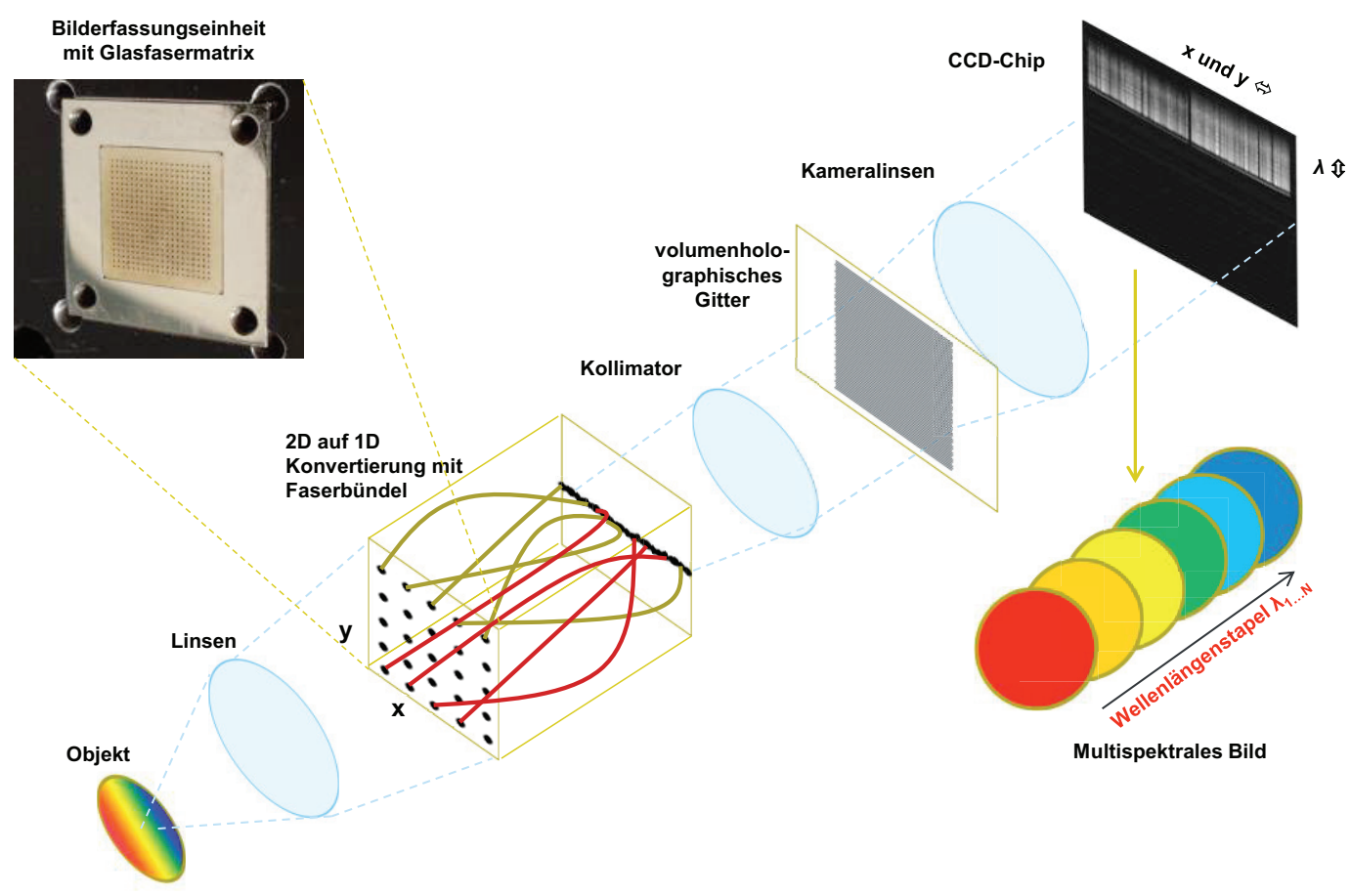

Abb. 1. Funktionsprinzip des Spektrographen.

Multimodefasern mit einer Kantenlänge von knapp $1 \mathrm{~cm}$. Auf der Spektrographenseite sind die Fasern des Bündels linear in einer Reihe angeordnet und bilden so quasi einen Eingangsspalt. Über ein volumenholographisches Gitter mit einer Beugungseffizienz von $80 \%$ wird das Lichtsignal auf einen großflächigen (4096 × 4112 Pixel) stickstoffgekühlten CCDChip gelenkt. Jede einzelne Faser erzeugt auf dem CCD-Chip eine individuelle, spektral abhängige Lichtspur. Eine eigens entwickelte Software „p3d“ ermittelt die Spektren aus dem CCD-Rohsignal und ordnet sie den korrespondierenden Bildpunkten an der Bilderfassungseinheit zu. Zu Speicherung und weiteren Verarbeitung wird letztendlich ein Datenkubus erzeugt. Eine Übertragung dieses Messprinzips in die Ramanspektroskopie wurde bereits unter verschiedenen Bezeichnungen vorgestellt [3].
Allerdings haben bisher beschriebene Versuchsanordnungen deutlich weniger Bildpunkte und sind auf die Untersuchung von sehr kleinen Proben im $\mu \mathrm{m}$-Bereich ausgelegt. Für die Identifizierung von krebsverdächtigen Hautpartien müssen aber Flächen von einigen Quadratmillimetern untersucht werden [1]. Abb. 2 zeigt einen Versuchsaufbau, der es ermöglicht, Ramanbilder von quadratischen Flächen mit einer Kantenlänge von 3,6 mm aufzunehmen. Eine Kombination aus zwei achromatischen Dubletts mit Infrarot-Antireflexionsbeschichtung projiziert das Objektbild, d.h. strenggenommen dessen Ramansignale, auf die Stirnfläche der Bilderfassungseinheit. Ein $785 \mathrm{~nm}$ Langpasskantenfilter dient zur Ausblendung des Anregungslasers und der Rayleighstreuung. 


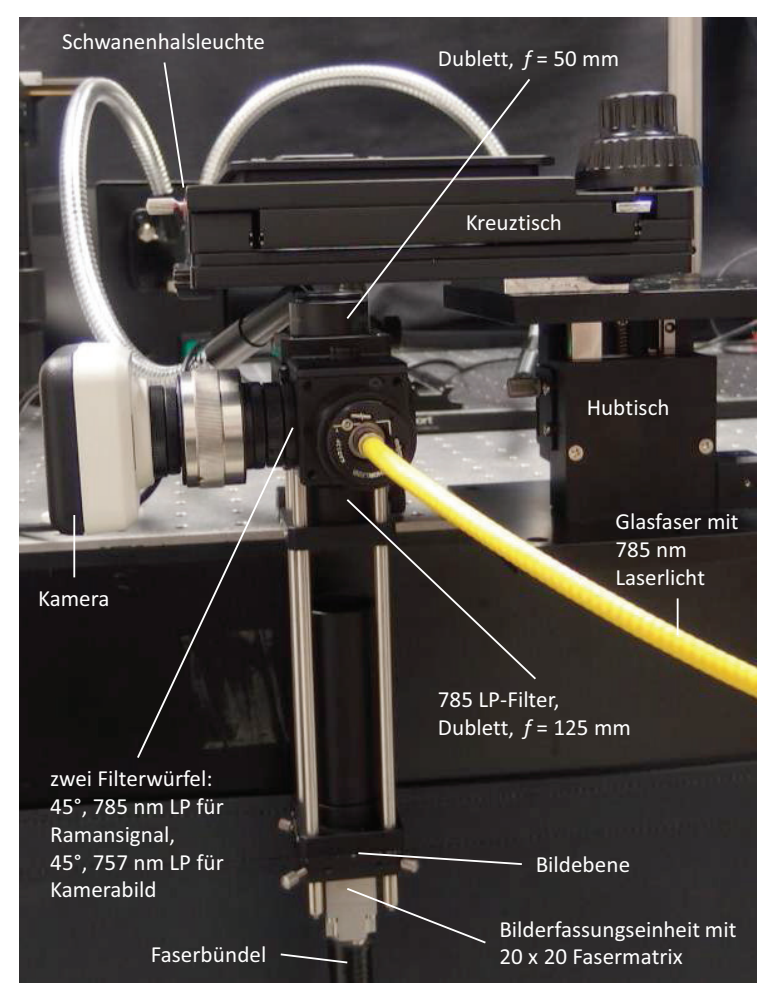

Abb. 2. Optikaufbau.

Über das Faserbündel wird das Licht zum Spektrographen geführt. Das Anregungslaserlicht wird über eine Glasfaser zugeführt und über einen dichroitischen $45^{\circ}$-Spiegel auf die Probe gelenkt. In der Ebene, in der sich die Probe befindet, ergibt sich ein gleichmäßiger ausgeleuchteter quadratischer Anregungslichtfleck mit einer Kantenlänge von 4,1 mm (Abb. 3).

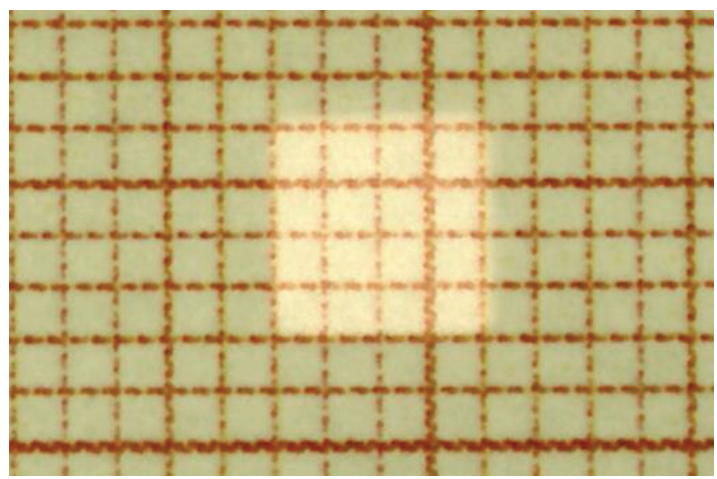

Abb. 3. Anregungslichtfleck auf Millimeterpapier in der Probenebene.

Im Strahlengang des Anregungslasers befindet sich ein weiterer dichroitischer Spiegel, der sichtbares Licht der Probe auskoppelt und auf eine Kamera lenkt, welche zur Positionierung und Fokussierung der Probe verwendet wird. Als Lichtquelle wird eine fasergekoppelte Duallaserlichtquelle LS-2-7878-FC (Newport, USA) verwendet, welche zwei umschaltbare Diodenlaser mit $784,5 \mathrm{~nm}$ und $785,5 \mathrm{~nm}$ Wellenlänge enthält. Die Laserleistungen wurden so eingestellt, dass in der Probenebene jeweils $230 \mathrm{~mW}$ erzielt werden, was einer Leistungsdichte von $14 \mathrm{~mW} / \mathrm{mm}^{2}$ entspricht. Es ist anzumerken, dass diese Leistungsdichte um zwei Größenordnungen geringer ist als bei vergleichbaren Einkanal-Ramanmikroskopen, die die gesamte Laserenergie mit einem Objektiv auf einen kleinen Punkt fokussieren. Entsprechend geringer sind bei dem hier vorgestellten Versuchsaufbau die Intensitäten der entstehenden Ramansignale. Zur Positionierung der Probe wird ein Kreuztisch verwendet, der zur Fokussierung wiederum mit einem Hubtisch verbunden ist. Auf diese Weise wird ein Versuchsaufbau erhalten, der einem inversen Fluoreszenzmikroskop ähnelt. Auf dem Kreuztisch kann eine Probenhalterung mit einer Bodenplatte aus Kalziumfluorid positioniert werden. Kalziumfluorid ist ein gut geeignetes Objektträgermaterial, da es selbst nur ein geringes Ramansignal generiert und damit wenig Untergrund verursacht.

\section{SERDS-Messungen}

Hintergrundfluoreszenz stellt eine besondere Herausforderung in der Ramanspektroskopie dar. Speziell die von biologischen Proben ausgehende Fluoreszenz kann selbst bei $785 \mathrm{~nm}$ Anregungslichtwellenlänge die Intensität der Ramansignale um Größenordnungen übersteigen. SERDS (Shifted Excitation Raman Difference Spectroscopy) ist eine Methode, den störenden Einfluss von Untergrundfluoreszenz auszublenden [7]. Dabei wird die Probe zweimal hintereinander mit leicht verschiedenen Anregungswellenlängen vermessen (d.h. mit der hier verwendeten Duallaserquelle bei 784,5 und $785,5 \mathrm{~nm}$ ). Die Methode basiert auf der Tatsache, dass Ramansignale immer relativ zur Anregungswellenlänge auftreten, Fluoreszenzsignale dagegen immer bei fixen Wellenlängen. Durch geeignete Differenzbildung und Transformation lässt sich das Ramanspektrum der Probe untergrundfrei rekonstruieren. Voraussetzung ist, dass sich die Probe während der Messung nicht verändert. Der hier verwendete Algorithmus ist in [8] beschrieben.

\section{Proben}

Die Leistungsfähigkeit des Versuchsaufbaus wurde an folgenden Proben demonstriert: „Aspirin Effekt“-Schmerzmittel (1), $10 €$-Banknote (2), Querschnitt durch ein Schweineohr (3), Schweinehaut (4). 


\section{Ergebnisse}

(1) Aspirin Effekt

Aspirin Effekt ist ein Schmerzmittelgranulat. Gemäß Beipackzettel enthält es neben dem Wirkstoff Acetylsalicylsäure noch D-Mannitol, Natriumhydrogencarbonat, Natriumdihydrogencitrat, Ascorbinsäure, Zitronensäure, Aromastoffe und Aspertam. Eine Spatelspitze des Granulats wurde auf einen $1 \mathrm{~mm}$ dicken Kalziumfluorid-Objektträger gegeben und fokussiert. Für jeweils $1 \mathrm{~s}$ wurden bei 784,5 und $785,5 \mathrm{~nm}$ die Ramanspektren aufgenommen. Abb. 4 zeigt das Kamerabild, die erhaltenen SERDS-Spektren an zwei ausgewählten Bildpunkten und die resultierende Verteilung der Acetylsalicylsäure in Falschfarben. Als Maßgröße wurde die Intensität der Ramanlinie bei $1.606 \mathrm{~cm}^{-1}$ verwendet.
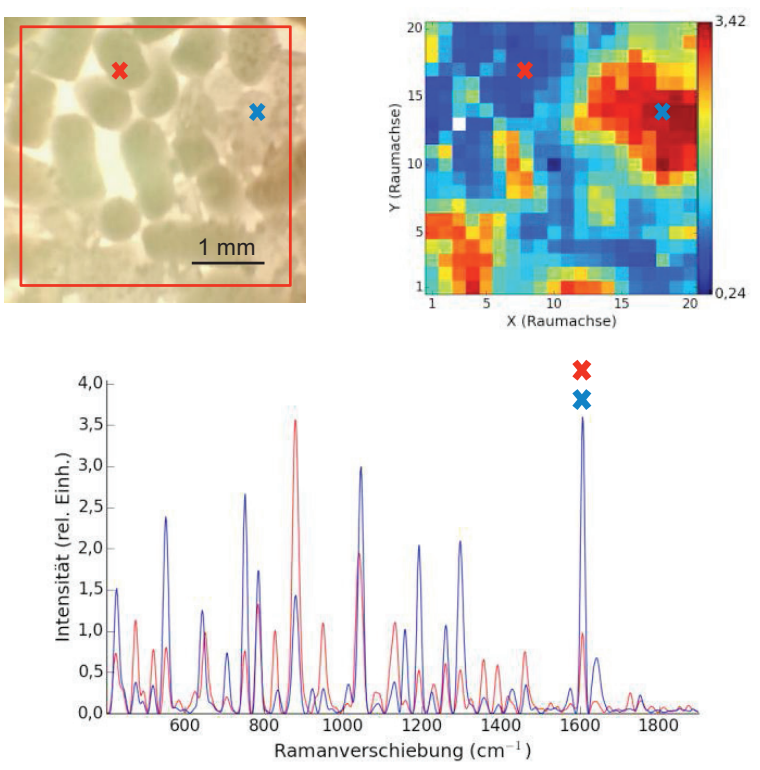

Abb. 4. „Aspirin Effekt"-Granulat. Links oben: Kamerabild. Rechts oben: Verteilung des Wirkstoffes Acetylsalicylsäure anhand der Ramanlinie bei $1.606 \mathrm{~cm}^{-1}$. Unten: SERDS-Spektren an den Stellen $*$ und $*$. Aufnahmezeit: 1 s pro Laserwellenlänge.

Offensichtlich ist der Wirkstoff Acetylsalicylsäure in Form eines feinen Pulvers vorhanden. Die walzenförmigen Granulatbestandteile scheinen keinen Wirkstoff zu enthalten.

\section{(2) $10 €$-Banknote}

Ein $10 €$-Geldschein wurde auf einen $1 \mathrm{~mm}$ Kalziumfluorid-Objektträger gelegt. Ein Teil des Buchstaben E des „EURO“-Schriftzuges auf der Vorderseite wurde fokussiert. Für jeweils $10 \mathrm{~s}$ wurden bei 784,5 und $785,5 \mathrm{~nm}$ die Ramanspektren aufgenommen. Abb. 4 zeigt das $\mathrm{Ka}$ merabild, Ramanspektren an zwei ausgewähl- ten Bildpunkten, von denen einer innerhalb und einer außerhalb des E's liegt. Die Druckfarbe des E's generiert charakteristische Ramanlinien. Die Intensität der Linie bei $1.567 \mathrm{~cm}^{-1}$ wurde als Maßgröße für das Falschfarbenbild verwendet.
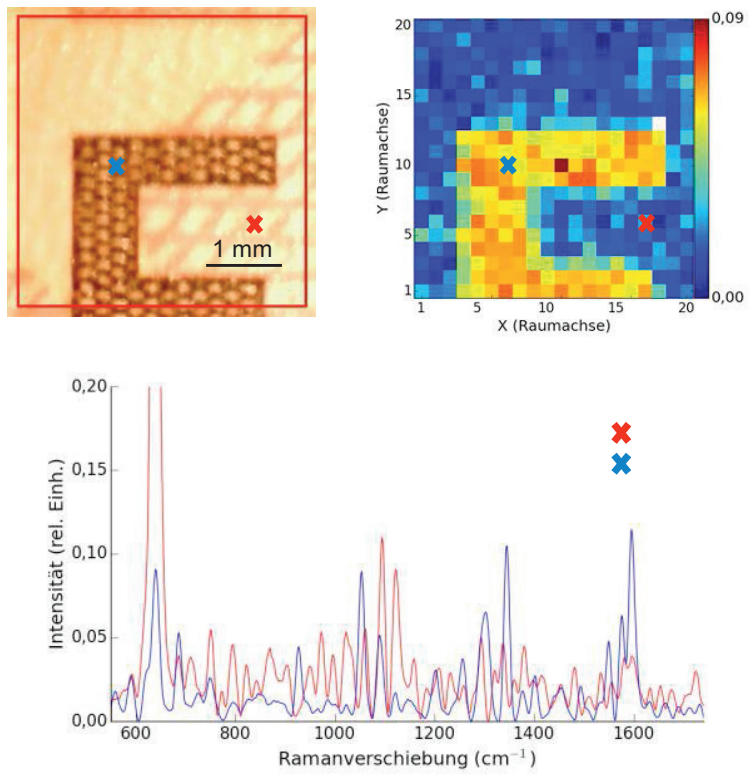

Abb. 5. Teil eines E's auf einem $10 €$-Schein. Links oben: Kamerabild. Rechts oben: Verteilung der Druckfarbe des E's anhand der Ramanlinie bei $1.567 \mathrm{~cm}^{-1}$. Unten: SERDSSpektren an den Stellen * und * Aufnahmezeit: 10 s pro Laserwellenlänge.

\section{(3) Querschnitt durch ein Schweineohr}

Von einem frischen Schweineohr wurde mit einem Skalpell ein Stück abgeschnitten, mit der Schnittfläche nach unten auf die Kalziumfluoridplatte eines Probengefäßes gestellt (Abb. 6). Die Schnittfläche wurde im Kamerabild fokussiert.

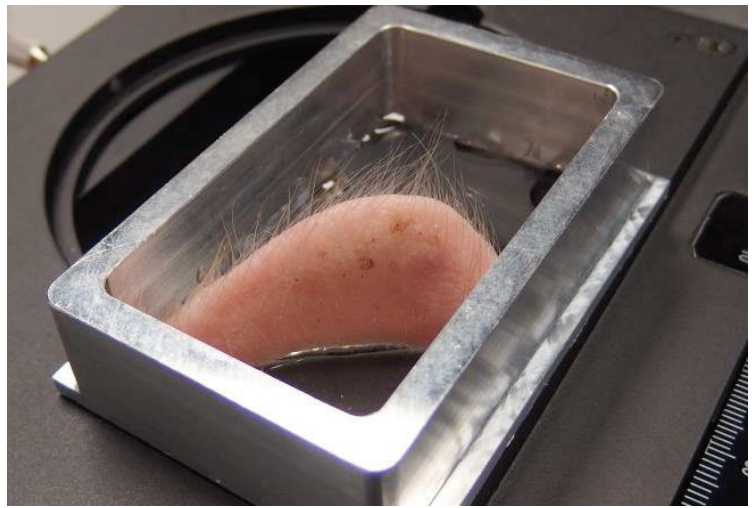

Abb.6. Schweineohr in Probengefäß. 
In der Mitte der Schnittfläche befindet sich augenscheinlich Fettgewebe. Im Kamerabild ist es als breiter, heller, leicht geneigter, vertikaler Streifen zu erkennen (Abb. 7).
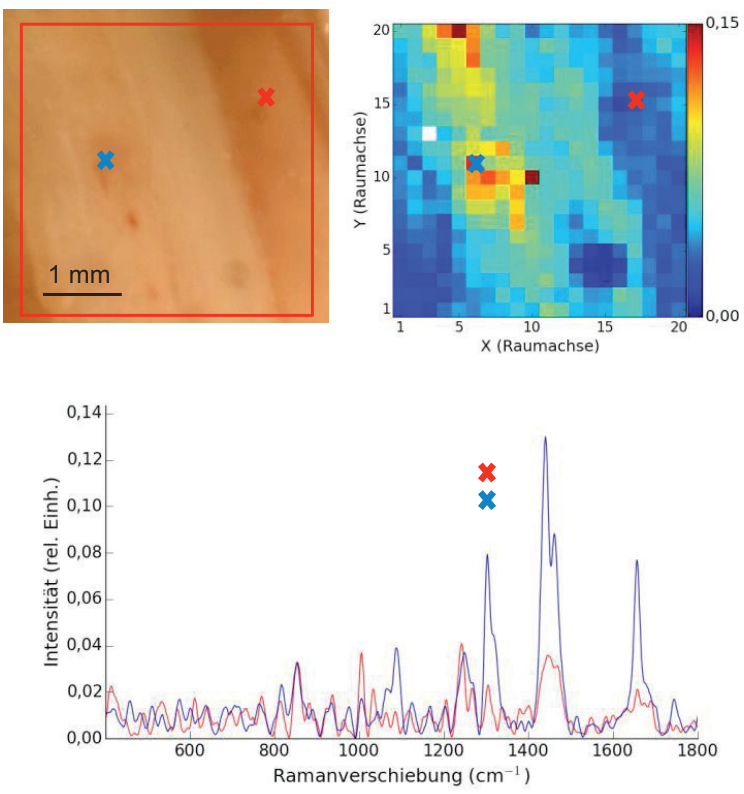

Abb. 7. Schnittfläche eines Schweineohrs. Links oben: Kamerabild. Rechts oben: Ramanbild anhand der Linie bei $1.303 \mathrm{~cm}^{-1}$. Unten: SERDS-Spektren an den Stellen * und * Aufnahmezeit: 60 s pro Laserwellenlänge.

Im Bereich der (X/Y)-Positionen (7/10) und (9/5) sind rötliche Flecken zu erkennen, vermutlich Blutreste. Bei (16/3) ist eine dunklere Stelle sichtbar. Das Ramanbild (Abb.7), welches anhand der Linie bei $1.303 \mathrm{~cm}^{-1}$ erstellt wurde, zeigt diese Positionen als rote bzw. dunkelblaue Falschfarbe. Die Aufnahmezeit pro Laserwellenlänge betrug jeweils $60 \mathrm{~s}$.

\section{(4) Schweinehaut}

Ein Stück Schweinehaut wurde in den Probenbehälter mit der Hautoberfläche nach unten auf die Kalziumfluoridplatte des Probengefäßes gelegt und fokussiert. Um einer eventuellen Austrocknung während des Messvorgangs vorzubeugen, wurde etwas Wasser auf den Boden des Probenbehälters gegeben und der Behälter mit einem Deckel verschlossen. Die Aufnahmezeit pro Laserwellenlänge betrug jeweils $60 \mathrm{~s}$. Das Ramanbild wurde anhand der Intensität der Ramanlinie bei $1.455 \mathrm{~cm}^{-1}$ erstellt.
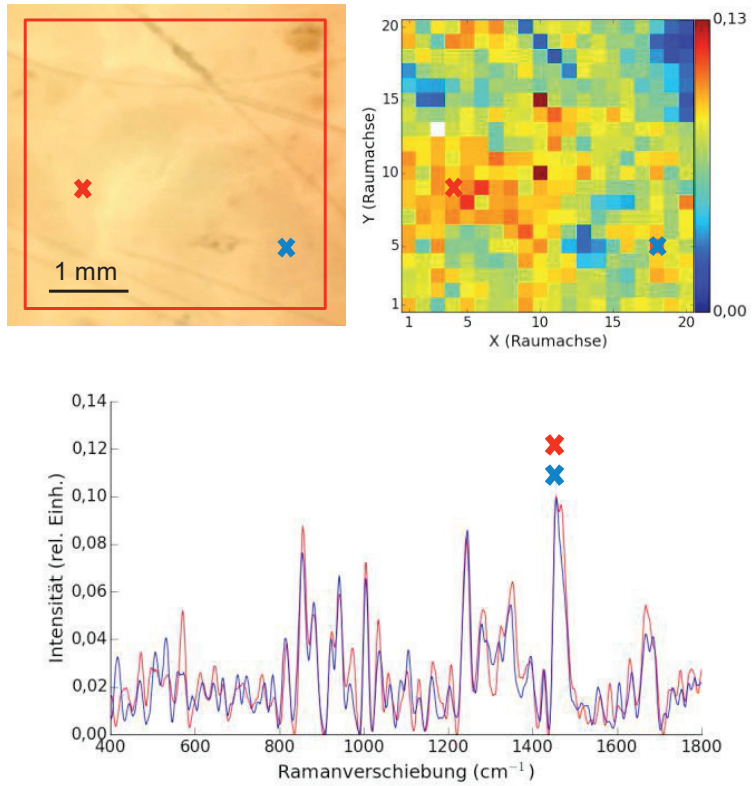

Abb. 8. Schweinhaut. Links oben: Kamerabild. Rechts oben: Ramanbild anhand der Linie bei $1.455 \mathrm{~cm}^{-1}$. Unten: SERDS-Spektren an den Stellen * und *. Aufnahmezeit: $60 \mathrm{~s}$ pro Laserwellenlänge.

Die im Kamerabild erkennbaren Strukturen finden sich im Ramanbild erstaunlich detailliert wieder. Gut erkennbar ist die Pigmentierung im der rechten oberen Ecke und das offenbar etwas verschmutzte Haarstück, das schräg durch das obere Viertel des Bildes läuft. Im Kamerabild schwächer erkennbare Pigmentierungen bei (3/15) und bei (13/5) finden sich ebenfalls im Ramanbild wieder.

\section{Schlussfolgerung}

Mit dem hier vorgestellten Versuchsaufbau konnten ortsaufgelöste Ramanbilder von großflächigen Proben $\left(13 \mathrm{~mm}^{2}\right)$ in einem einzigen Belichtungsvorgang aufgenommen werden. Die Messzeiten für das komplette Bild betrugen lediglich 120 s (Schweineohr, Schweinehaut), 20 s (10 €-Banknote) und $2 \mathrm{~s}$ (Aspirin Effekt), was bei 400 Bildpunkten Messzeiten von 300, 50 bzw. $2 \mathrm{~ms}$ pro Bildpunkt entspricht. Selbst bei der einfachen Analysemethode, die reine Intensität bei einer bestimmten Ramanverschiebung in Falschfarben darzustellen, korrespondieren die Ramanbilder kontrastreich zu den im Kamerabild erkennbaren Strukturen. Dieses Ergebnis stimmt zuversichtlich, im nächsten Schritt Krebsgewebe mit einer komplexeren Clusteranalyse identifizieren zu können. 


\section{Literaturangaben}

[1] C. Reble, I. Gersonde, C. Dressler, J. Helfmann, W. Kühn, H.J. Eichler, Evaluation of Raman spectroscopic macro raster scans of native cervical cone biopsies using histopathological mapping, Journal of Biomedical Optics 19, 027007 (2014); doi: 10.1117/1.JBO.19.2.027007

[2] L. Opilik, T. Schmid, R. Zenobi, Modern Raman Imaging: Vibrational Spectroscopy on the Micrometer and Nanometer Scales, Annu. Rev. Anal. Chem. 6, 379-398 (2013); doi: 10.1146/annurev-anchem-062012-092646

[3] S. Stewart, R.J. Priore, M.P. Nelson, P.J. Treado, Raman Imaging, Annu. Rev. Anal. Chem. 5, 337260 (2012); doi: 10.1146/annurev-anchem062011-143152

[4] http://www.aip.de/de/aktuelles/presse/muse Zugriff am 07.10.2015

[5] E. Schmälzlin, M. Stolz, B. Moralejo, S. Adelhelm, M.M. Roth, Imaging multiplex Raman spectroscopy with no need for sequential scanning, Laser+Photonics 62-65 (2015)

[6] E. Schmälzlin, B. Moralejo, M. Rutowska, A. Monreal-Ibero, C. Sandin, N. Tarcea, J. Popp, M.M. Roth, Raman Imaging with a Fiber-Coupled Multichannel Spectrograph, Sensors 14, 2196821980 (2014); doi:10.3390/s141121968

[7] K. Sowoidnich, H.-D. Kronfeldt, Fluorescence Rejection by Shifted Excitation Raman Difference Spectroscopy at Multiple Wavelengths for the Investigation of Biological Samples, ISRN Spectroscopy 2012, Article ID 256326 (2012); doi: $10.5402 / 2012 / 256326$

[8] H. Schmidt, P. Kaiser, M. Maiwald, Europäische Patentschrift EP 2480868 B1, Patentblatt 2014/02

\section{Danksagung}

Wir danken Frau Dr. Gisela Thiede und Herrn Dr. Maxim Darvin von der Charité-Universitätsmedizin Berlin für die Bereitstellung von Proben. Wir danken dem Bundesministerium für Bildung und Forschung (BMBF) für die finanzielle Unterstützung des Forschungsvorhaben (VIP-Programm 03V0843). 